\title{
Light-Matter Interactions in Aligned Silver Nanorod Arrays
}

Md Aman Uddin and Ugur Pasaogullari

Department of Mechanical Engineering, Center for Clean Energy Engineering, University of Connecticut, Storrs, CT 06269, USA

Corresponding Author:

Md Aman Uddin

Center for Clean Energy Engineering

University of Connecticut

44 Weaver Road, Storrs, CT, 06269 USA

Phone: (860) 617-9409

Fax: (860) 486-5088

E-mail: aman.uddin@engr.uconn.edu 


\begin{abstract}
This study presents an experimental evaluation of the light-matter interactions in silver nanorod arrays of various lengths fabricated by electron-beam physical vapor deposition. The reflectance of nanorod arrays is found to decrease with increasing nanorod length, reaches to a minimum and then again increases with increasing length. Since nanorod arrays are subwavelength structures, the decrease in reflectance is primarily due to surface plasmon resonance. At the tip of the nanorods, the light excites the surface plasmons, which propagate along the conductor-dielectric interface. Hence, longer and consequently higher surface area nanorod arrays provide more dielectric-conductor interface for light to couple with the surface plasmons and more surface to scatter resulting in lower reflectance.
\end{abstract}

Keywords: Silver nanorod, Physical vapor deposition, Reflectance, Surface plasmon resonance 


\section{Introduction}

Physical vapor deposition (PVD) grown silver (Ag) nanorods have attracted significant attention recently in science and technological applications, including plasmonics $[1,2]$, and sensors [3-6] through surface-enhanced Raman spectroscopy (SERS). Such nanorods have shown SERS enhancement factors of $\sim 10^{9}$ [7] and this enhancement strongly depends on the length of the nanorods [3], the incident angle [7], the polarization of excitation light [8], and the design of the structure [9].

Zhao et al. [7] extensively worked on PVD grown Ag nanorods and characterized the optical properties of nanorods grown at various deposition angles and lengths. They reported that SERS enhancement factor increases nearly monotonically with the decrease of the reflectance of $\mathrm{Ag}$ nanorods at the SERS excitation wavelength, and the reflectance depends on the length of the Ag nanorods as well as on the deposition angle. They also reported that Ag nanorods deposited with larger deposition angles provide lower reflectance. It is also reported that zig-zag structure of Ag nanorod arrays enhance SERS performance, possibly by creating hot spots for increased SERS at the bends [9]; but there is a lack in clear understanding of the mechanism behind the optical behavior of PVD grown Ag nanorods.

In this study, we report the optical reflectance of Ag nanorod arrays with varying length of nanorods at a fixed high deposition angle $\left(86.5^{\circ}\right)$. We also report, for the first time, optical properties of zig-zag structure of Ag nanorod arrays. We found that long and high surface area nanorod arrays show lower reflectance, which may be attributed to the surface plasmon resonance. 


\section{Methods}

Ag nanorods are fabricated by the oblique angle deposition using a custom designed high vacuum electron beam PVD. The source material is Ag pellets (99.99\%, Kurt J. Lesker Co., Jefferson Hills, PA, USA) and is placed in a graphite liner at the base of the vacuum chamber. The vacuum chamber is approximately $45 \mathrm{~cm}$ tall and $25 \mathrm{~cm}$ in diameter. The $\mathrm{Ag}$ nanorods are grown on Si $\{100\}$ substrates (Nova Electronic Materials, Flower Mound, TX, USA) with native oxide layer. First, Si substrates are ultrasonically cleaned using acetone, ethanol, and deionized water and then placed at the top of the chamber. A base layer of $500 \mathrm{~nm} \mathrm{Ag} \mathrm{film} \mathrm{is} \mathrm{deposited} \mathrm{at} \mathrm{a}$ low deposition rate of $0.5 \mathrm{~nm} / \mathrm{s}$ to form a uniform thickness film. Then the substrates are oriented to an oblique angle of $86.5^{\circ}$ using a custom designed precision mount. The source to substrate distance is approximately $30 \mathrm{~cm}$. The Ag nanorods are grown to a thickness of 50, 100, 250, 500, 750 , and $1000 \mathrm{~nm}$ at a deposition rate of $2 \mathrm{~nm} / \mathrm{s}$. The deposition is monitored by a quartz crystal microbalance (QCM) positioned perpendicular to the incoming flux. In each deposition, base pressure was around $6.7 \times 10^{-4} \mathrm{~Pa}$.

Immediately following the deposition, Ag nanorods are analyzed using scanning electron microscope (SEM) (FEI Quanta 250, FEI, Hillsboro, OR, USA) with an accelerating voltage of $10 \mathrm{kV}$, and energy dispersive X-ray spectroscopy (EDX) with an accelerating voltage of $20 \mathrm{kV}$. In order to identify crystallographic structure, $\mathrm{Ag}$ nanorods arrays are characterized by X-ray diffraction (XRD) using a Bruker D8 Advanced system with $\mathrm{Cu} K \alpha$ radiation $(\lambda=1.5406 \AA)$. A sample of Ag nanorod arrays on Si substrate with dimension $1.5 \mathrm{~cm} \times 1.5 \mathrm{~cm}$ is used for XRD analysis. The reflectance of the Ag nanorod arrays is measured using a UV-VIS 2600 spectrometer with an integrating sphere attachment (Shimadzu Corporation, Kyoto, Japan). Ag nanorods are fabricated in high vacuum condition and are stored in vacuum chamber between 
analyses, however nanorods are exposed to environment during sample preparation for SEM, and during UV-VIS measurements and a thin oxide layer may form at the surface.

To measure surface area of each nanorod arrays, high resolution images are analyzed. From multiple locations with area of $1 \mu \mathrm{m}^{2}$ of high resolution images, average length and average diameter of the nanorods are measured and number density is counted manually. Although the nanorods are not entirely cylindrical and uniform or smooth, approximate total surface area in $1 \mu \mathrm{m}^{2}$ base area is calculated assuming all the nanorods are cylindrical with the average measured diameter. The parameters of Ag nanorod arrays with the length of 50 and $1000 \mathrm{~nm}$ are not presented here because nanorods are too short to measure in $50 \mathrm{~nm}$ nanorod arrays, and nanorods are overlapped each other in $1000 \mathrm{~nm}$ nanorod arrays.

\section{Results and Discussion}

Figure 1 shows SEM images of the nanorod arrays fabricated at $86.5^{\circ}$ with three different lengths. The nanorod arrays are grown due to so called geometric shadowing effect [10, 11], and the actual lengths of the nanorods are larger than QCM measured values (Table 1). At smaller lengths, nominal diameters of the nanorods are small (Table 1) and they are well separated. With increasing length, nanorods start to overlap each other, resulting in a decreased number density and increased diameter of the nanorods. As the length of the nanorods increase, total surface area increases and reaches to a maximum as long as nanorods remain separated, and decreases when nanorods start to merge together (Figure 2). Under the current deposition conditions, highest surface area is found for $500 \mathrm{~nm}$ length Ag nanorod array.

The nanorods are characterized using EDX and XRD to analyze the chemical compositions and crystallographic structure, respectively. Here, we present the results of Ag nanorod arrays with $500 \mathrm{~nm}$ as an example, however all samples show similar chemical composition and 
crystallographic structure. EDX (Figure 3a) shows the presence of Ag on the Si wafer, and XRD pattern (Figure 3b) shows the polycrystalline behavior of the Ag nanorod arrays. XRD pattern contains peaks at $2 \theta=38.11,44.30,64.45,77.40$, and 81.54 which are indexed as (111), (200), (220), (311), and (222) planes, respectively, which correspond to face-centered cubic Ag. The film has a higher intensity of (111) crystal orientation similar to what is reported by Khare et al. [12] and Oh et al. [13].

Figure 4 shows the reflectance of the base Ag layer and Ag nanorod arrays of different lengths. There is no difference observed in the reflectance profile of 500 and $2000 \mathrm{~nm}$ thick base $\mathrm{Ag}$ films, therefore the reflectance measured on $500 \mathrm{~nm} \mathrm{Ag}$ film shows the characteristics of $\mathrm{Ag}$ and, only reflectance of $500 \mathrm{~nm} \mathrm{Ag}$ film is shown. In wavelengths $>400 \mathrm{~nm}$, average $\mathrm{Ag}$ reflectance is $96 \%$ which shows highly reflecting nature of Ag. At $\sim 320 \mathrm{~nm}$ wavelength, there is a sharp drop in reflectance which is attributed to the interband absorption associated either with transitions from Fermi level to the next higher empty band or with transitions from a lower lying filled band to the Fermi level [14]. There is also a drop in reflectance at $225 \mathrm{~nm}$ which can also be attributed to interband transition [14].

To eliminate the effect of substrate in the reflectance measurements, nanorod arrays are grown on $500 \mathrm{~nm} \mathrm{Ag} \mathrm{film.} \mathrm{Reflectance} \mathrm{profiles} \mathrm{show} \mathrm{similar} \mathrm{pattern} \mathrm{for} \mathrm{all} \mathrm{nanorod} \mathrm{arrays.}$ There are drops in reflectance at 320 and $225 \mathrm{~nm}$ similar to Ag base layer. In wavelength $>400 \mathrm{~nm}$, reflectance decreases with an increase in the length of nanorods, reaches a minimum and then starts to increases. As shown in Figure 5 for the wavelengths of 600 and $1200 \mathrm{~nm}$, the minimum reflectance is found for $500 \mathrm{~nm} \mathrm{Ag} \mathrm{nanorod} \mathrm{arrays,} \mathrm{which} \mathrm{has} \mathrm{the} \mathrm{highest} \mathrm{surface} \mathrm{area.}$ The change in the reflectance with the length of the Ag nanorods is proportional to the total surface area, and the high surface area nanorod arrays show lower reflectance. 
Along with overall decrease in the reflectance, there are some other specific drops (valleys) at certain wavelength in the reflectance profile listed in Table 2. These drops and overall decrease in reflectance can be attributed to the surface plasmon (SP) resonance absorption [14], which is defined as a collective motion of electrons excited by the lights and travel along the dielectric/conductor interface and hence decrease reflectance [15]. The dimensions and gaps between Ag nanorods are well below the optical wavelength. In these subwavelength structures, light can couple to SPs at the nanorod tips and SPs propagate along the nanorods, allowing them to serve as optical waveguides [16]. Barbara et al. [16] reported that 760 to $860 \mathrm{~nm}$ wavelengths of light can travel 11 to $17.5 \mu \mathrm{m}$ in $\mathrm{Ag}$ nanowire with a diameter of $80 \mathrm{~nm}$. Light propagates along the nanorods (through SPs) and scatter inside the nano-structure and can be absorbed by neighboring nanorods. In nanorods arrays with high surface area, there is a larger surface area for light interaction with SPs and more light is absorbed resulting in lower reflectance.

Moreover, SP induced light absorption is believed to be enhanced by the nanoscale topologies, such as small gaps, corners, or bends, named hot spots [9]. In order to generate potential hot spots by bending nanorods, a zigzag Ag structure with small corners is fabricated. First, $\mathrm{Ag}$ nanorods are grown in one direction and the substrate is rotated $180^{\circ}$, and nanorods are grown again in the alternating direction, and zigzag structure is obtained by rotating the substrate $180^{\circ}$ several times while depositing the nanorods. Figure 6a shows the SEM image of zigzag structure with two foldings of the Ag nanorod. At each step before the rotation, the QCM measured length of the Ag nanorods was $250 \mathrm{~nm}$. The reflectance is measured at each step and presented in Figure 6b. With one folding, reflectance decreases compared to the one layer of Ag nanorod arrays showing confinement of light in the subwavelength structures. With two foldings, 
reflectance does not decrease further, but increases slightly due to decrease in overall porosity of the structure, consequently lower surface area.

It is necessary to mention here that optical properties of the aligned Ag nanorod arrays are anisotropic and they strongly depend on polarization of incident angle because when light hits aligned nanorods, the oscillation of the electric field induces the longitudinal plasmon mode along the nanorods and the transverse plasmon mode perpendicular to the nanorods $[8,17]$. Understanding the effect of the polarization of incident angle and individual components of surface plasmon resonance is part of our ongoing work.

\section{Summary and Conclusions}

Silver nanorod arrays are fabricated by oblique angle deposition in an electron-beam physical vapor deposition at a constant deposition angle at different lengths. Shorter nanorods grow with small diameter and they are well separated. With the increase of the length, nanorod diameter increases and neighboring nanorods overlap each other, decreasing the overall surface area. Larger nanorods fuse together and are no longer separated. The reflectance of the nanorod arrays is lower compared to uniform Ag film, and reaches to a minimum for the nanorod arrays with highest surface area.

Characteristic dimensions of the nanorod arrays are well below the wavelength of the light. In these subwavelength structures, the decrease in reflectance can be attributed to the surface plasmon resonance. High surface area nanorod arrays provides higher dielectric-conductor interface for light-matter coupling resulting in lower reflectance. 


\section{Acknowledgement}

The authors gratefully acknowledge financial support from the National Science Foundation (CBET-0748063). The authors also thank Dr. Stephen P Stagon for help with physical vapor deposition.

\section{References}

[1] P.C. Andersen, K.L. Rowlen, Brilliant optical properties of nanometric noble metal spheres, rods, and aperture arrays, Appl. Spectrosc. 56 (2002) 124A-124A.

[2] P. Bartlett, J. Baumberg, S. Coyle, M. Abdelsalam, Optical properties of nanostructured metal films, Faraday Discuss. 125 (2004) 117-132.

[3] S.B. Chaney, S. Shanmukh, R.A. Dluhy, Y. Zhao, Aligned silver nanorod arrays produce high sensitivity surface-enhanced Raman spectroscopy substrates, Appl. Phys. Lett. 87 (2005) 031908.

[4] S. Shanmukh, L. Jones, J. Driskell, Y. Zhao, R. Dluhy, R.A. Tripp, Rapid and sensitive detection of respiratory virus molecular signatures using a silver nanorod array SERS substrate, Nano Lett. 6 (2006) 2630-2636.

[5] X. Sun, S. Stagon, H. Huang, J. Chen, Y. Lei, Functionalized aligned silver nanorod arrays for glucose sensing through surface enhanced Raman scattering, RSC Advances 4 (2014) $23382-23388$.

[6] H.Y. Chung, C.C. Chen, P.C. Wu, M.L. Tseng, W.C. Lin, C.W. Chen, H.P. Chiang, Enhanced sensitivity of surface plasmon resonance phase-interrogation biosensor by using oblique deposited silver nanorods, Nano. Res. Lett. 9 (2014) 476. 
[7] Y. Liu, H.Y. Chu, Y. Zhao, Silver nanorod array substrates fabricated by oblique angle deposition: morphological, optical, and SERS characterizations, J. Phy. Chem. C. 114 (2010) 8176-8183.

[8] Y. Zhao, S. Chaney, Z. Zhang, Absorbance spectra of aligned Ag nanorod arrays prepared by oblique angle deposition, J. Appl. Phys. 100 (2006) 063527.

[9] Q. Zhou, X. Zhang, Y. Huang, Z. Li, Y. Zhao, Z. Zhang, Enhanced surface-enhanced Raman scattering performance by folding silver nanorods, Appl. Phys. Lett. 100 (2012) 113101.

[10] L. Abelmann, C. Lodder, Oblique evaporation and surface diffusion, Thin Solid Films 305 (1997) 1-21.

[11] H. van Kranenburg, C. Lodder, Tailoring growth and local composition by obliqueincidence deposition: a review and new experimental data, Mater. Sci. Eng. R: Reports. 11 (1994) 295-354.

[12] C. Khare, C. Patzig, J. Gerlach, B. Rauschenbach, B. Fuhrmann, Influence of substrate temperature on glancing angle deposited Ag nanorods, J. Vac. Sci. Technol. A. 28 (2010) 1002-1009.

[13] M.K. Oh, Y.S. Shin, C.L. Lee, R. De, H. Kang, N.E. Yu, B.H. Kim, J.K. Yang, Morphological and SERS properties of silver nanorod array films fabricated by oblique thermal evaporation at various substrate temperatures, Nano. Res. Lett. 10 (2015) 259.

[14] Y. Lu, H. Zhang, F. Liu, UV-visible reflectance spectra of nanocrystalline silver compacted under different pressures, Phy. Lett. A. 342 (2005) 351-356.

[15] R. Hillenbrand, T. Taubner, F. Keilmann, Phonon-enhanced light-matter interaction at the nanometre scale, Nature. 418 (2002) 159-162. 
[16] B. Wild, L. Cao, Y. Sun, B.P. Khanal, E.R. Zubarev, S.K. Gray, N. F. Scherer, M. Pelton, Propagation lengths and group velocities of plasmons in chemically synthesized gold and silver nanowires, ACS Nano. 6 (2012) 472-482.

[17] Y. Jen, W. Liu, J. Chao, J. Huang, Y. Chang, Strong light coupling effect for a glancingdeposited silver nanorod array in the Kretschmann configuration, Nano. Res. Lett. 9 (2014) 597. 


\section{List of Figures}

Fig. 1. Shape of nanorod arrays at different lengths. SEM images of Ag nanorod arrays with length (a) $100 \mathrm{~nm}$, (b) $500 \mathrm{~nm}$, and (c) $750 \mathrm{~nm}$.

Fig. 2. Total surface area of different Ag nanorod arrays.

Fig. 3. (a) Energy dispersive X-ray spectroscopy (EDX) and (b) X-ray diffraction (XRD) pattern of Ag nanorod arrays (500 $\mathrm{nm}$ length) on silicon wafer.

Fig. 4. Reflectance of Ag nanorod (NR) arrays with different lengths. All the nanorods are grown on $500 \mathrm{~nm}$ thick flat Ag base layer.

Fig. 5. Reflectance of Ag nanorod at different wavelengths (300, 600, and $1200 \mathrm{~nm})$ as a function of nanorod length.

Fig. 6. (a) SEM image and (b) reflectance profile of zigzag Ag nanostructures. 


\section{List of Tables}

Table 1 Parameters of Ag nanorod arrays.

Table 2 Reflectance valley wavelength of Ag nanorod arrays. 
Table 1 Parameters of Ag nanorod arrays

\begin{tabular}{|l|l|l|l|l|}
\hline Length of & Actual & Nanorod & Average & Total surface \\
nanorod & average & density \\
QCM (nm) & nanorod & number/ $\left.\mu \mathrm{m}^{2}\right)$ & $\begin{array}{l}\text { area in } 1 \mu \mathrm{m}^{2} \\
\text { diameter } \\
(\mathrm{nm})\end{array}$ & \\
\hline Base layer & - & $(\mathrm{nm})$ & - & $1 \mu \mathrm{m}^{2}$ \\
\hline 100 & $140 \pm 20$ & $100 \pm 5$ & $46 \pm 20$ & $3.19 \mu \mathrm{m}^{2}$ \\
\hline 250 & $298 \pm 20$ & $47 \pm 5$ & $74 \pm 20$ & $5.41 \mu \mathrm{m}^{2}$ \\
\hline 500 & $540 \pm 20$ & $32 \pm 5$ & $96 \pm 20$ & $7.80 \mu \mathrm{m}^{2}$ \\
\hline 750 & $800 \pm 20$ & $13 \pm 5$ & $150 \pm 20$ & $6.11 \mu \mathrm{m}^{2}$ \\
\hline
\end{tabular}


Table 2 Reflectance valley wavelength of Ag nanorod arrays.

\begin{tabular}{|c|c|}
\hline $\begin{array}{c}\text { Ag nanorod } \\
\text { length (nm) }\end{array}$ & $\begin{array}{c}\text { Reflectance valley } \\
\text { wavelength (nm) }\end{array}$ \\
\hline 50 & 360 \\
\hline 100 & $355,485,585$ \\
\hline 250 & 490,590 \\
\hline 500 & $355,485,590$ \\
\hline 750 & $355,485,590$ \\
\hline 1000 & \\
\hline
\end{tabular}



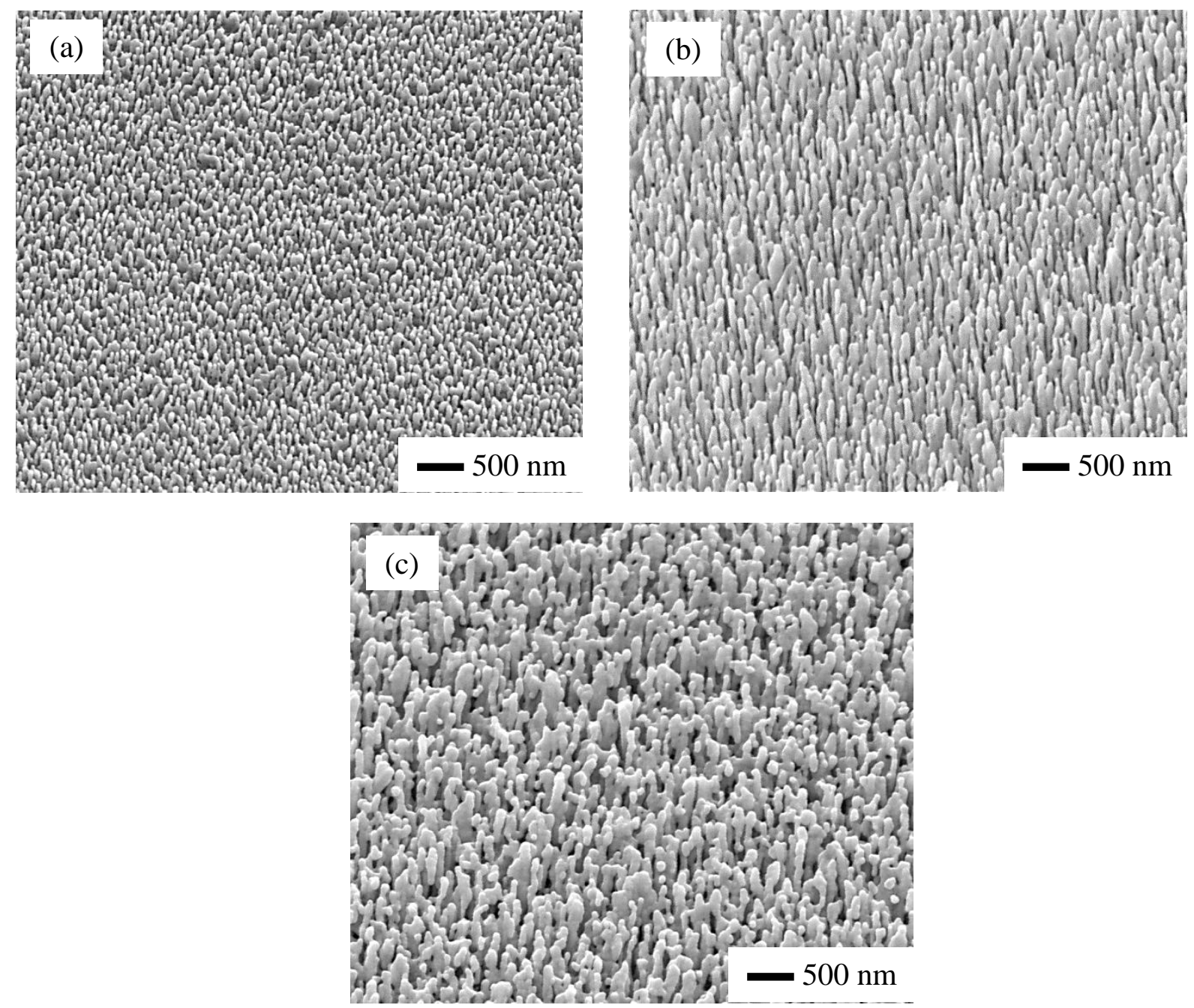

Fig. 1. Shape of nanorod arrays at different lengths. SEM images of Ag nanorod arrays with length (a) $100 \mathrm{~nm}$, (b) $500 \mathrm{~nm}$, and (c) $750 \mathrm{~nm}$. 


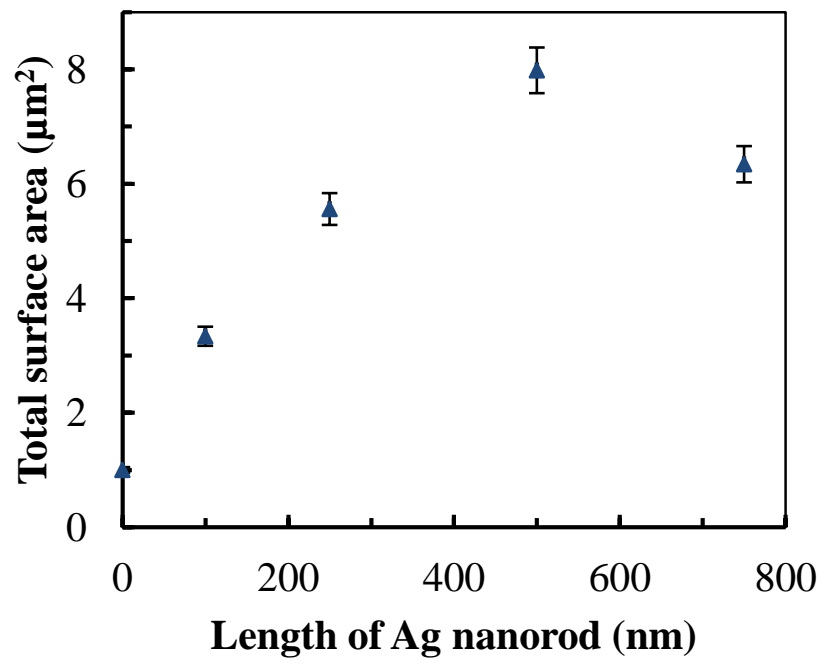

Fig. 2. Total surface area of different Ag nanorod arrays. 

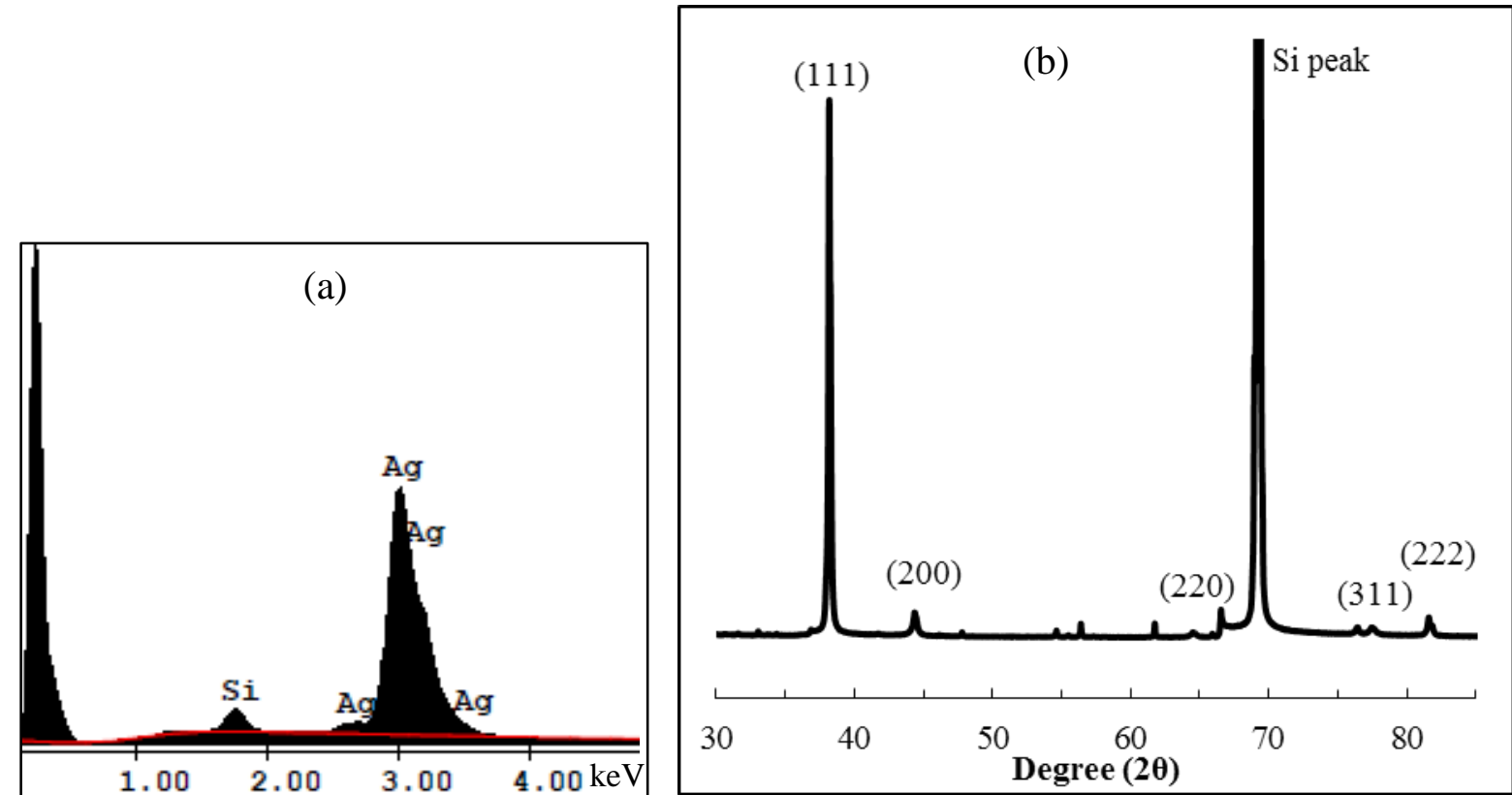

Fig. 3. (a) Energy dispersive X-ray spectroscopy (EDX) and (b) X-ray diffraction (XRD) pattern of Ag nanorod arrays (500 nm length) on silicon wafer. 

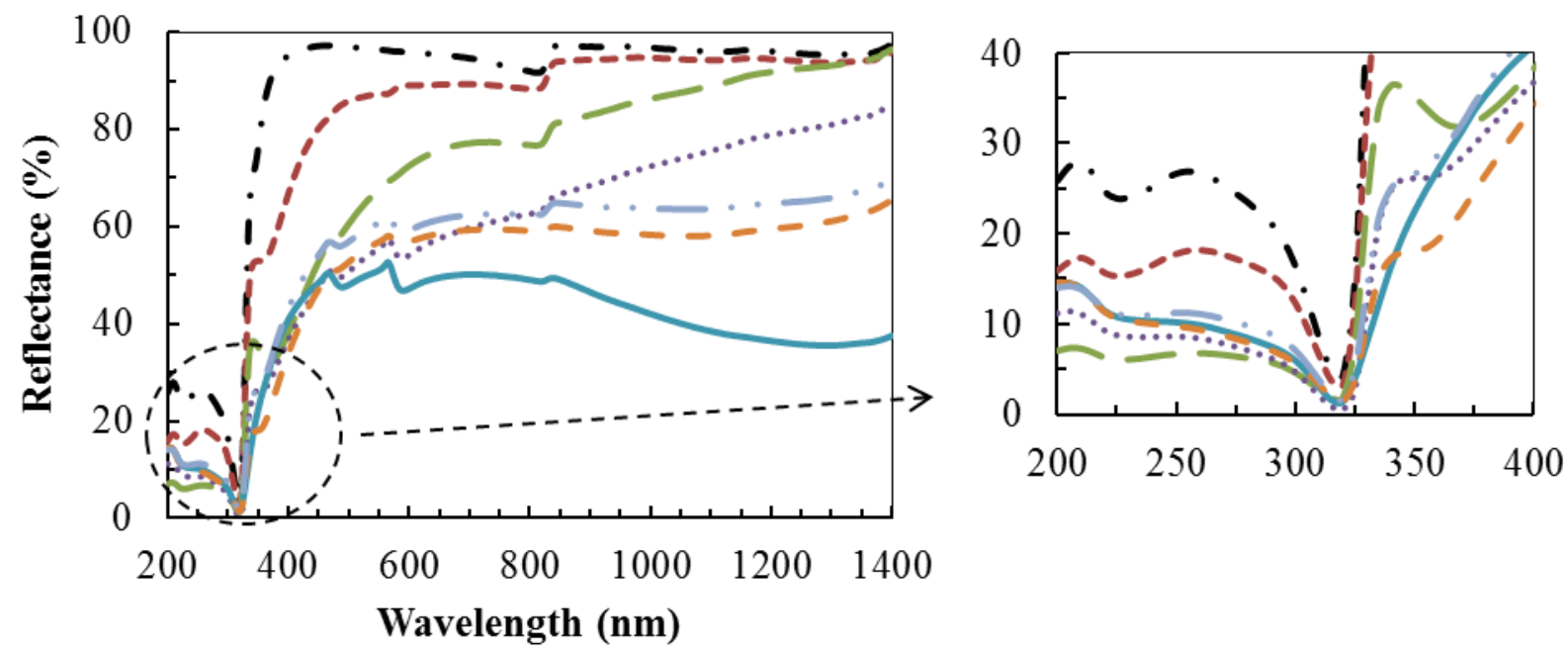

- - Ag base layer --- $-50 \mathrm{~nm} \mathrm{Ag} \mathrm{NR \quad -}-100 \mathrm{~nm} \mathrm{Ag} \mathrm{NR}$ $250 \mathrm{~nm}$ Ag NR $500 \mathrm{~nm} \mathrm{Ag} \mathrm{NR} \quad-\quad 750 \mathrm{~nm} \mathrm{Ag} \mathrm{NR} \quad-.1000 \mathrm{~nm} \mathrm{Ag} \mathrm{NR}$

Fig. 4. Reflectance of Ag nanorod (NR) arrays with different lengths. All the nanorods are grown on $500 \mathrm{~nm}$ thick flat Ag base layer. 


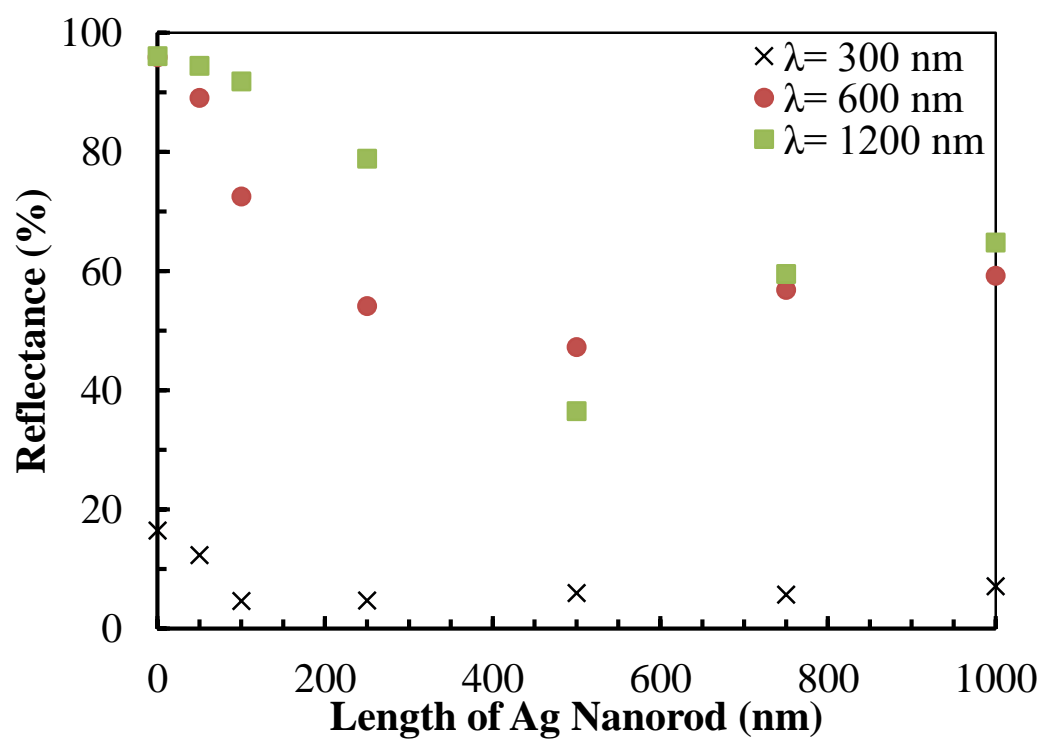

Fig. 5. Reflectance of Ag nanorod at different wavelengths ( $300 \mathrm{~nm}, 600 \mathrm{~nm}$, and $1200 \mathrm{~nm}$ ) as a function of nanorod length. 
(a)

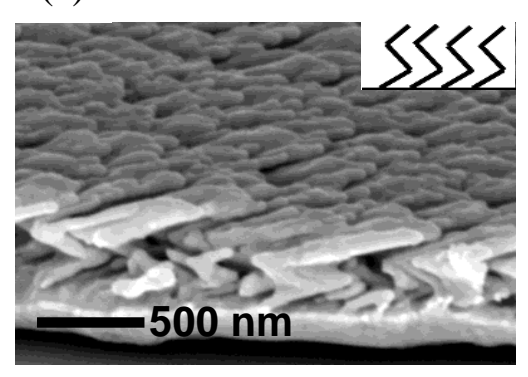

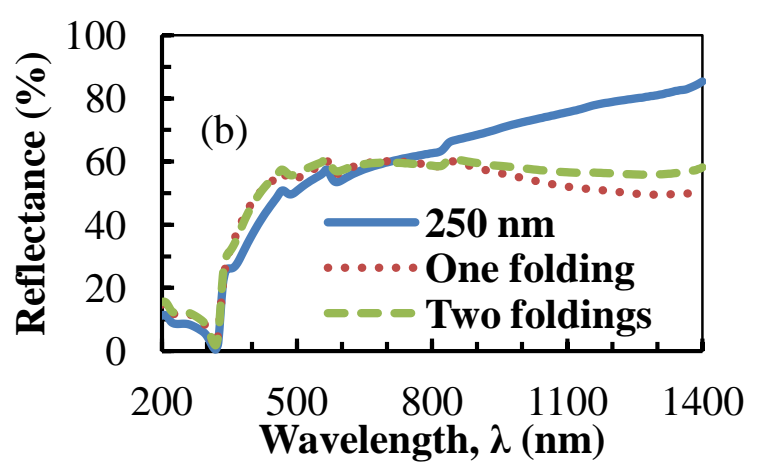

Fig. 6. (a) SEM image and (b) reflectance profile of zigzag Ag nanostructures. 